\title{
Two-dimensional Self-assembly of a Designed Amphiphilic Peptide at Air/Water Interface
}

\author{
By Masayoshi TANAKA,${ }^{1}$ Kempei OGURA, ${ }^{1}$ Souhei ABIKO,${ }^{1}$ \\ Naokiyo KOSHIKAWA, ${ }^{2}$ and Takatoshi KINOSHITA ${ }^{1, *}$
}

\begin{abstract}
A peptide having alternate sequence of hydrophobic and hydrophilic amino acid residues, $(\mathrm{QAQL})_{4}\left(\mathrm{CH}_{3} \mathrm{CO}-(\mathrm{Gln}\right.$-Ala-GlnLeu $)_{4}-\mathrm{NH}_{2}$ ), was designed to form a $\beta$-sheet monolayer at the air/water interface. According to the designing of the peptide having charge free amino acid as hydrophilic residues, the peptide showed stable monolayer at wide range $\mathrm{pH}$. The peptide monolayer prepared by Langmuir-Blodgett (LB) method showed an arranged nano-fibrous object. (QAQL) 4 -PEG was also an attractive building block for construction of nano-architecture by self-assembly at the air/water interface. PEG attached on the peptide segment showed a conformational transition from a flattened state to brush one, dependent on the compressing degree of the monolayer on water subphase. The conformational transition was simultaneously induced a morphological change of the nano-architecture from disk-like structure to fibrous array.

KEY WORDS: Self-assembly / Air/water Interface / PEG-attached Peptide / $\beta$-Sheet Fiber / Nano-architecture /
\end{abstract}

The functional surface having a regulated morphology has a number of potential applications. ${ }^{1-5}$ The ordered structure is explored to create architecture in submicro/nano-meter scale, which could be applied for electro-devices, biointerfaces and super-hydrophilic/hydrophobic materials and so on. In order to construct a well-ordered structure on the material surface, two principal methods are suggested. One is "top-down" approach such as lithography and etching, which can construct a well-defined architecture at sub-micron scale. ${ }^{6,7}$ The other is "bottom-up" approach, which would be able to construct various nano-architectures, such as nano-fibers, nano-tubes and vesicles etc. ${ }^{8,9}$ In these decades, molecular self-assembly has been a most promising technique to create such a fine-structure in nano-meter scale.

Peptides and proteins are useful building block for construction of nano-structured materials, since the character of each amino acid and its sequence promote to a favorable secondary structure such as $\alpha$-helix, $\beta$-sheet and flexible loop. These structural units show inter/intra-molecular interaction, and thus resulting in specific higher-ordered nano-organizations. ${ }^{10}$ Many researchers are making efforts to exploit the selfassembly of peptides and proteins. The formed nano-architectures such as micelle, gel and film, have versatile application in the field of drug-delivery, tissue-engineering and surface science. ${ }^{11,12}$ On the other hand, two-dimensional self-assembled structures are also attractive by virtue of the regulated structures such as stripe, lattice and honeycomb. ${ }^{13,14}$ These nano-architectures would be applied as a template for electro device and surface modification.

We have focused on the construction of self-assembled structure of peptide at the air/water interface. The LB technique is an advanced method for preparing ordered organic monolayer, and it is expected to lead a designing of novel nano-device system. ${ }^{15}$ When the peptide has an amphiphilic sequence, the phase direction of the peptide monolayer would be controlled due to the amphiphilic nature of the air/water interface. Furthermore, the structure of the constructed monolayer would be modulated by fixing various external factors such as $\mathrm{pH}$ of aqueous subphase and compressing degree. Previously, we have reported that RFDF16 $\left(\mathrm{CH}_{3} \mathrm{CO}-(\mathrm{Arg}-\mathrm{Phe}-\right.$ Asp-Phe $)_{4}-\mathrm{NH}_{2}$ ), which formed $\beta$-sheet conformation due to its amphiphilic binary sequence, self-assembled into a fibrous structure at the air/water interface to give an arranged nanostripe. ${ }^{16}$ Since the hydrophilic residues of RFDF16 were composed of an equivalent number of cationic and anionic residues, the balance of the electrostatic interaction was dominated by $\mathrm{pH}$ of the water subphase. Thus, once the electrostatic repulsion was superior in two-dimensional selfassembly of RFDF16, the regulation of the fiber array would be destabilized. In this study, we have designed an amphiphilic peptide, (QAQL) ${ }_{4}$ composed of charge free amino acid as hydrophilic residue. The peptide would form stable twodimensional self-assembled architecture at various $\mathrm{pH}$, since the peptide is free from electrostatic repulsion. Two-dimensional self-assembly at the air/water interface was investigated by the amphiphilic peptide. A corresponding peptide moiety obtained by attaching poly(ethylene glycol)(PEG) at C terminus, (QAQL) $)_{4}$-PEG, was also investigated to form two-dimensional nanostructure at the air/water interface.

\section{EXPERIMENTS}

\section{Materials}

The amphiphilic peptides were prepared by solid phase method using 9-fluorenylmethoxycarbonyl (Fmoc) strategy. The peptide chain was synthesized on CLEAR-Amide resin

\footnotetext{
${ }^{1}$ Department of Frontier Materials, Graduate School of Engineering, Nagoya Institute of Technology, Gokiso-cho, Showa-ku, Nagoya 466-8555, Japan 2Japan Aerospace Exploration Agency, 2-1-1 Sengen, Tsukuba 305-8505, Japan

*To whom correspondence should be addressed (Tel: +81-52-735-5267, Fax: +81-52-735-5267, E-mail: kinoshita.takatoshi@ @itech.ac.jp).
} 
(Peptide Institute. Inc.) by using 3 equivalents of Fmoc-amino acid derivatives. The activation and coupling reactions have been conducted using 3 equivalent of 1-hydroxy-7-benzotriazole (HOAt), and 3 equivalent of 1,3-diisopropylcarbodiimide (DIPCDI) in $\mathrm{N}, \mathrm{N}$-dimethylformamide (DMF). And $20 \%$ of piperidine was used in DMF for removal of Fmoc group. The $N$-terminus of (QAQL) 4 was acetylated by 10 equivalent of acetic anhydride in DMF on the resin. To cleave the peptides from the resin and deprotection of the side chain were carried out with trifluoroacetic acid (TFA)/1,2-ethanedithiol/thioanisole/water (84:8:4:4). The peptides were precipitated and washed by diethylether.

(QAQL) $)_{4}$-PEG was prepared by same procedure with TentaGel PAP resin (RAPP Polymere) instead of CLEARAmide resin. Molecular weight of PEG chain preloaded on the TentaGel PAP resin is 3,000. Cleavage process was followed by the above procedure.

\section{Measurement}

The peptide dissolved in 2,2,2-trifluoroethanol(TFE) was diluted by milliQ water to be $2.6 \times 10^{-6} \mathrm{~mol} \mathrm{dm}^{-3}$ including $10 \%$ TFE. The secondary structure of peptide was investigated by circular dichroism (CD) spectroscopy. CD spectra were measured by J-820K spectropolarimeter (JASCO Ltd.) under a nitrogen atmosphere. Experiments were performed in a quartz cell with a $5 \mathrm{~mm}$ path length over the range of $190-260 \mathrm{~nm}$ at room temperature.

\section{Self-assembly at the Air/water Interface}

Surface pressure-area $(\pi$-A) isotherms of RFDF16 were measured using a NL-BIO40-MWCT (Nippon Laser \& Electronics Lab.) at $25^{\circ} \mathrm{C}$. Monolayers were prepared by spreading a solution of the peptide in benzene/2,2,2-trifluoroethanol (6:4) at a concentration of approximately $0.1 \mathrm{mg} / \mathrm{mL}$ through a microsyringe onto deionized water surface. After evaporation of organic solvents at $20 \mathrm{~min}$, the monolayer was compressed at a speed of $5 \mathrm{~mm} / \mathrm{min}$. Surface pressure was measured by Wilhelmy plate. The monolayer was transferred onto a freshly cleaved mica substrate in vertical method (upstroke) at a speed of $5 \mathrm{~mm} / \mathrm{min}$ after the surface pressure was reached at targeted pressure and then the monolayer was stabilized for $20 \mathrm{~min}$. The subphase $\mathrm{pH}$ was controlled by addition of $\mathrm{HCl}$ for acidic condition and $\mathrm{NaOH}$ for alkaline condition.

\section{RESULTS AND DISCUSSIONS}

\section{Self-assembly Behavior of $(\mathrm{QAQL})_{4}$ at the Air/water Interface}

We have designed a peptide having alternative sequence of hydrophobic and hydrophilic amino acid, $\left(\mathrm{CH}_{3} \mathrm{CO}-(\mathrm{QAQL})_{4}-\right.$ $\mathrm{NH}_{2}$ ). It is composed of glutamine $(\mathrm{Q})$ as a hydrophilic moiety and alanine (A), leucine (L) as hydrophobic moieties (Scheme 1). It is well known that peptide having alternative sequence of hydrophobic and hydrophilic amino acid tends to take a $\beta$-sheet conformation. When (QAQL) 4 took a $\beta$-sheet
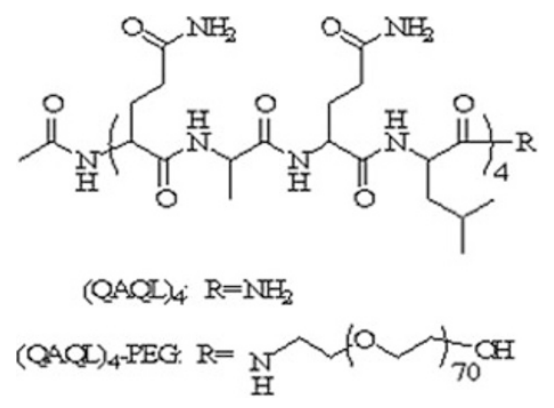

Scheme 1. Structure of the peptides.

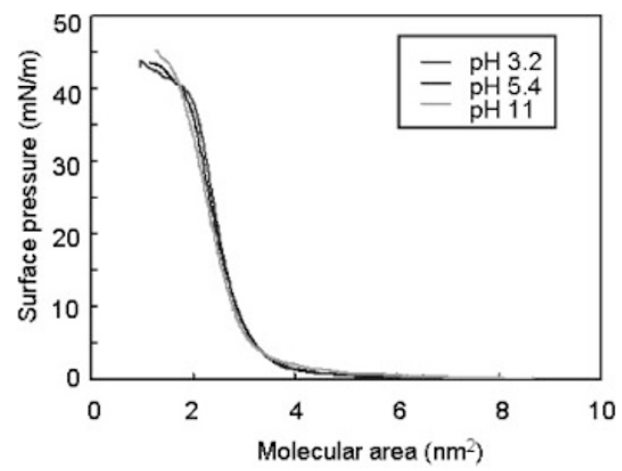

Figure 1. Surface pressure-area $(\pi-A)$ isotherm for monolayer of $(Q A Q L)_{4}$ at the air/water interface $(\mathrm{pH}=3.2,5.4$, and 11).

structure, the hydrophilic and hydrophobic phase should face in the opposite side, and the peptide would turn the hydrophilic side to aqueous subphase at the air/water interface.

$\pi-\mathrm{A}$ isotherm of (QAQL) 4 on MilliQ water ( $\mathrm{pH} 5.4$ ) was investigated. The surface pressure was increased at a certain area by compressing the spread peptide layer (Figure 1). The obtained curve was typical as a formation of monolayer, thus, it was suggested that (QAQL) 4 formed a monolayer at the air/water interface. From the $\pi$-A isotherm, limiting area per molecule was estimated to be $3.0 \mathrm{~nm}^{2}$. The limiting area was in reasonable agreement with the calculated value $\left(2.8 \mathrm{~nm}^{2}\right)$ on the basis of $\beta$-sheet peptide with 16 residues. The peptide monolayer was investigated on the water subphase in acidic ( $\mathrm{pH}$ 3.2) and alkaline condition $\mathrm{pH} 11.0)$. $\pi$-A isotherms at each $\mathrm{pH}$ were successfully obtained, and the limiting area per molecule were estimated to be $3.0 \mathrm{~nm}^{2}$ both for $\mathrm{pH} 3.2$ and 11 . The values were agreed with the obtained value of the peptide monolayer on the MilliQ water. In the previous report, electrostatic repulsion on the side chain of the hydrophilic amino acid was influenced on the self-assembly behavior to give several isotherms by $\mathrm{pH}$ change. ${ }^{16}$ It was suggested that $(\mathrm{QAQL})_{4}$ enables the effective construction of two-dimensional nano-architecture by Langmuir technique due to the insensitive characteristics to the $\mathrm{pH}$ change.

In order to verify the self-assembled structure of the peptide at the air/water interface, Langmuir-Blodgett (LB) film was constructed. The monolayer constructed at the higher compressing degree often shows no difference of altitude affecting with the densely packed structure of the peptides. Thus, the LB 

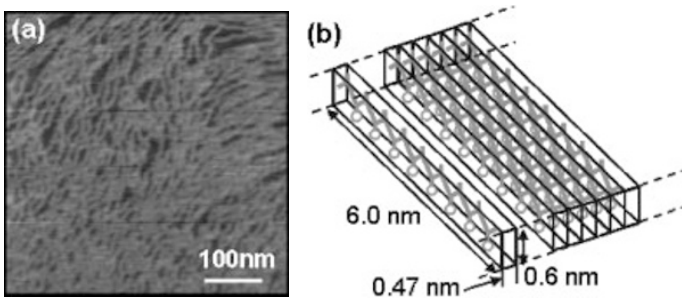

Figure 2. (a) AFM image of the obtained LB film of $(Q A Q L)_{4}$. The film was transferred on mica substrate without compressing process at $\mathrm{pH}$ 5.4. (b) Schematic illustration of the formed fibrous object of $(\mathrm{QAQL})_{4}$ on the water subphase.

film was constructed at the surface pressure where the peptides adopt weakly interaction, i.e., $\pi=1.4 \mathrm{mN} / \mathrm{m}\left(\mathrm{A}=4.0 \mathrm{~nm}^{2}\right)$. The surface morphology of the LB film on a mica substrate was investigated by AFM (Figure 2a). AFM image of the obtained LB film showed fibrous object arranged in two-dimension. The width and thickness of the obtained fibrous object were estimated to be $6.0-8.0 \mathrm{~nm}$ and $0.4-0.6 \mathrm{~nm}$, respectively. The theoretical size of width and thickness, when the peptide took $\beta$-sheet conformation are $5.5 \mathrm{~nm}$ and $0.6 \mathrm{~nm}$, respectively (Figure $2 \mathrm{~b}$ ). The values in the observed image were reasonably agreed with the theoretical values, suggesting that the fibrous object was attributed to the self-assembled structure of $\beta$-sheet peptide.

\section{Self-assembly Behavior of (QAQL) $)_{4}$-PEG at the Air/water Interface}

Two-dimensional self-assembly behavior of (QAQL) $)_{4}$-PEG was also investigated on MilliQ water subphase $(\mathrm{pH}=5.4)$. The obtained isotherm showed a quasi-plateau region which was characteristic behavior of PEG segment at the air/water interface (Figure 3). At low surface pressure, a typical behavior of liquid-expanded monolayer was observed. The peptide segment of (QAQL) $)_{4}$-PEG takes $\beta$-sheet structure facing hydrophilic residues to water subphase due to its binary sequence. PEG segment also remain at the air/water interface anchoring with the peptide segment, while PEG segment adopts a flattened conformation due to the affinity for water (Figure 3A). When the both segments exist at the air/water interface, two-dimensional micro-phase separation proceeds between peptide and PEG segment. The self-assembled monolayer was transferred at $\pi=8 \mathrm{mN} / \mathrm{m}$ on the freshly cleaved mica. AFM image of the obtained LB film was shown in Figure 4a. Disk-like aggregate having uniform thickness $(0.7 \mathrm{~nm})$ was observed as a major structure. The width of the observed aggregate was $6 \mathrm{~nm}$, which agreed with the peptide length in $\beta$-sheet conformation. The peptide adopting $\beta$-sheet structure on the water subphase assembled by inter-strand hydrogen bonding would form peptide domain. Due to the anchoring PEG segment, further formation of inter-strand hydrogen bonding would be interfered by steric hindrance. Such a disk-like structure, where hydrophobic and hydrophilic segment located on the core and periphery, respectively, is often obtained by phase separation of an amphiphilic diblock copolymer without particular compressing process. ${ }^{17,18}$ The
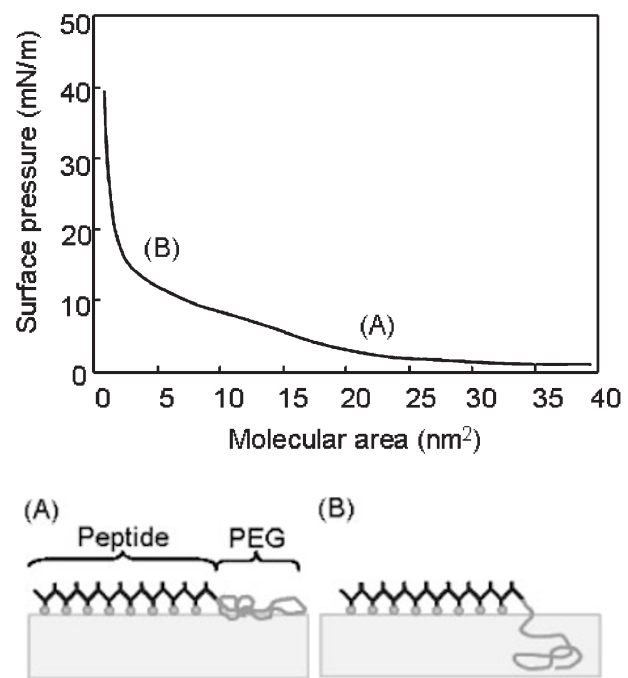

Figure 3. (a) $\pi$-A isotherm for monolayer of $(Q A Q L)_{4}-P E G$ at the air/water interface $(\mathrm{pH}=5.4)$, (b) Schematic illustration of the PEG location on the compressing of the monolayer; $A$ and $B$ represent before and after the pseudo plateau, respectively.
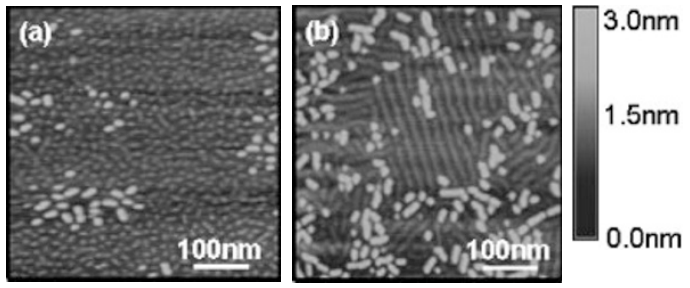

Figure 4. AFM images of the obtained $L B$ film of $(Q A Q L)_{4}$ transferred at (a) $8 \mathrm{mN} / \mathrm{m}$, and (b) $10 \mathrm{mN} / \mathrm{m}$.

phase separated structure in disk-like shape is one of the most stable structure in two-dimensional self-assembly based on the amphiphilicity. It is worthy of note that the constructed structure was successfully obtained in nano-meter scale, since the hydrophobic domain was composed of $\beta$-sheet peptide of 16 residues. Disk-like aggregate having larger thickness $(1.5 \mathrm{~nm})$ was also observed in the AFM image as minor structure. The side chain of glutamine could form hydrogen bonding to give a vertical aggregation on the water subphase.

As increasing the surface pressure at $9 \mathrm{mN} / \mathrm{m}$, pseudo plateau was slightly observed in the isotherm. This behavior is associated with the dissolution of PEG segment into the water subphase. This pseudo plateau is usually observed in the selfassembly behavior of PEG homopolymer ${ }^{19}$ and block copolymer having PEG segment. ${ }^{20,21}$ At this surface pressure, conformation at the air/water interface is transformed from a flattened conformation in contact with water surface to a brush conformation where PEG segments are located in the water.

At high surface pressure, the isotherm showed a steep rise due to the high resistance to compression of peptide layer having the brush conformation. The limiting area per molecule estimated from the steep isotherm was $2.5 \mathrm{~nm}^{2}$. The theoretical value of the (QAQL) $)_{4}$-PEG in the flattened conformation and $(\mathrm{QAQL})_{4}$ is $9.9 \mathrm{~nm}^{2}$ and $2.8 \mathrm{~nm}^{2}$, respectively. The estimated 


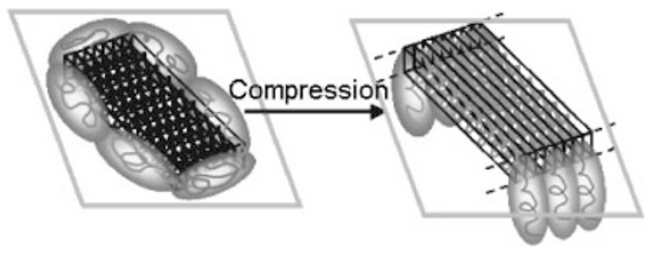

Figure 5. Schematic illustration of the phase-separated structure of $(\mathrm{QAQL})_{4}-\mathrm{PEG}$ at the air/water interface as compressing the monolayer.

value was close to the molecular area of the peptide segment, supporting that the steep rise in the isotherm attributed to the compression of the brush conformation (Figure 3B). The selfassembled monolayer was transferred at $\pi=10 \mathrm{mN} / \mathrm{m}$ on mica substrate. The fibrous objects aligned in two-dimension was observed in AFM image of the obtained LB film (Figure $4 \mathrm{~b}$ ). The fibrous object was assigned to be $\beta$-sheet structure, since the width of the fibrous object agreed with the length of the peptide in $\beta$-sheet conformation. It was obviously suggested that the propagation of $\beta$-sheet was proceeded by compressing the peptide-PEG layer. At the high surface pressure, the steric hindrance of PEG segment to the formation of inter-strand hydrogen bonding was reduced by transferring into brush conformation (Figure 5). The number of larger aggregates was increased at high surface pressure. As compressing the peptide monolayer, formation of hydrogen bonding at side chain of glutamine was induced, then the vertical aggregation would be promoted.

\section{CONCLUSION}

The construction of an arranged nano-stripe structure by an amphiphilic peptide designed to form a $\beta$-sheet was demonstrated. The peptide having alternate sequence of hydrophobic and hydrophilic amino acid residues spontaneously formed a nano-fibrous structure at the air/water interface. The selfassembling behavior was independent with $\mathrm{pH}$ of the water subphase, since the designed hydrophilic amino acid was composed of charge free residue. Attaching PEG on the $\beta$-sheet peptide was also attractive building block. A unique twodimensional segregated structure was constructed by the phase separation between $\beta$-sheet peptide and PEG segment. A conformational change from flattened to brush state was proceeded by compressing of the peptide-PEG monolayer. This behavior enabled morphological transition from disk-like aggregate to 2D array of nano-fibers. These results would provide a novel strategy for construction of two-dimensional nano-architectures.

Acknowledgment. A part of this work was performed as the "International space station applied research partnership program" of the Japan Aerospace Exploration Agency (JAXA) and the Nagoya Institute of Technology.

Received: July 8, 2008 Accepted: September 10, 2008 Published: October 22, 2008

\section{REFERENCES}

1. R. Blossey, Nat. Mater., 2, 301 (2003).

2. T. Nakanishi, N. Miyashita, T. Michinobu, Y. Wakayama, T. Tsuruoka, K. Ariga, and D. G. Kurth, J. Am. Chem. Soc., 128, 6328 (2006).

3. H. Rapaport, K. Kjaer, T. R. Jensen, L. Leiserwitz, and D. A. Tirrell, J. Am. Chem. Soc., 122, 12523 (2000).

4. H. Yokoi, S. Hayashi, and T. Kinoshita, Prog. Polym. Sci., 28, 341 (2003).

5. T. Ubukata, T. Seki, and K. Ichimura, Adv. Mater., 12, 1675 (2000).

6. M. L. Pang, J. Lim, J. Fu, R. B. Xing, C. X. Luo, and Y. C. Han, Opt. Mater., 23, 547 (2003).

7. A. Matsuo, M. Taki, S. A. Haque, Y. Yamamoto, T. Kikutani, S. Yamada, H. Nojiri, M. Motokawa, and H. Hori, Physca B, 298, 294 (2001).

8. J. D. Hartgerink, E. Beniash, and S. I. Stupp, Science, 294, 1684 (2001).

9. T. Shimizu, M. Masuda, and H. Minamikawa, Chem. Rev., 105, 1401 (2005).

10. T. Niwa, H. Yokoi, T. Kinoshita, and S. Zhang, Polym. J., 36, 665 (2004).

11. K. Osada and K. Kataoka, Adv. Polym. Sci., 202, 113 (2006).

12. S. Zhang, Nat. Biotechnol., 21, 1171 (2003).

13. E. T. Powers, S. I. Yang, C. M. Lieber, and J. W. Kelly, Angew. Chem., Int. Ed., 41, 127 (2002).

14. H. Yabu, M. Tanaka, K. Ijiro, and M. Shimomura, Langmuir, 19, 6297 (2003).

15. P. Yang, Nature, 425, 243 (2003).

16. M. Hattori, S. Hayashi, H. Yokoi, M. Tanaka, and T. Kinoshita, e-polymers, 31, 245 (2006).

17. J. K. Cox, K. Yu, B. Constantine, A. Eisenberg, and R. B. Lennox, Langmuir, 15, 7714 (1999).

18. J. Zhang, H. Cao, X. Wan, and Q. Zhou, Langmuir, 22, 6587 (2006)

19. D. J. Kuzmenka and S. Granick, Macromolecules, 21, 779 (1988).

20. A. M. Gonçalves da Silva and E. J. M. Filipe, Langmuir, 12, 6547 (1996).

21. T. J. Joncheray, K. M. Denoncourt, M. A. R. Meier, U. S. Schubert, and R. S. Duran, Langmuir, 23, 2423 (2007). 